using antibodies against the protein tyrosine phosphatase, CD45RO, and the chemokine receptor, CCR7. Tetramer tagged cells were magnetically enriched and analysed by flow cytometry.

Results We found a distinct population of Hcgp39-specific T cells in an RA patient at a frequency of $6 / 1,000,000$ CD4 T cells. Interestingly, Hcgp39-specific T cells can also be detected in healthy individuals. In one blood donor, 16/1,000,000 CD4 $\mathrm{T}$ cells are Hcgp39 tetramers positive. Initial immunophenotyping of these self-antigen specific $T$ cells indicates that almost the entire population is antigen-experienced in the RA patient (92\%). Unexpectedly, the majority of Hcgp39-specific population detected in the healthy person also has a memory phenotype (63\%), suggesting prior antigen exposure and T cell activation.

Conclusion Rare antigen-experienced Hcgp39-specific CD4 $T$ cells can be detected in the peripheral blood of RA patients and healthy individuals. Study is currently ongoing to evaluate functional differences between these autoreactive $T$ cell populations and to define additional self-epitopes recognised by CD4 T cells in healthy and disease states.

\section{A190 PROFILING THE T CELL REPERTOIRE IN RHEUMATOID ARTHRITIS}

L Su

10.1136/ard.2010.129668z1

Purpose Rheumatoid arthritis (RA) is one of the most common debilitating systemic inflammatory conditions. To date, little is known about the autoantigens involved in RA and how $\mathrm{T}$ cells recognising self-proteins may become pathogenic in disease. The aim of this study is to determine whether autoreactive $T$ cells in RA patients are different from those that can be found in healthy individuals.

Method Autoantigen-specific T cells are identified directly ex vivo using peptide-MHC tetramers. HLA-DR4 monomers with a tethered thrombin cleavable CLIP peptide were purified from the culture supernatant of a stable HLA-DR4 transfectant cell line by antibody affinity chromatography. The purified monomers were then biotinylated, thrombin cleaved, loaded with peptides, and oligomerised onto a streptavidin backbone labeled with a fluorophore. To identify individuals carrying the HLA-DRB1*0401 (DR4) allele, HLA typing was performed on healthy blood donors and RA patients using sequence-specific primer PCR. Individuals with DR4 allele(s) were selected for $\mathrm{T}$ cell repertoire analysis. To identify antigen-specific T cells, blood from DR4 positive individuals were enriched for CD4 cells by rosettasep/ficoll centrifugation. Tetramer staining was performed at room temperature for one hour using $10 \mathrm{nM}$ of tetramers loaded with peptides from hemagglutinin (HA) and the human glycoprotein 39 (Hcgp39). Memory phenotyping was performed 